\title{
Comparative Analysis of Resonant Converters for Energy Storage Systems
}

\author{
Stoyan Vuchev ${ }^{{ }^{*}}$, Dimitar Arnaudov ${ }^{1}$, and Nikolay Hinov ${ }^{1}$ \\ ${ }^{1}$ Technical university of Sofia, Faculty of Electronic Engineering and Technologies, Department of Power electronics, 1000 Sofia, \\ Bulgaria
}

\begin{abstract}
The following paperwork presents a comparative analysis of multiphase resonant converters for applications in energy storage systems. Models of the examined converters are developed in the software environments of MATLAB and LTspice. Results from the simulation examination of the converters during charging of supercapacitors and rechargeable batteries are presented. These results are compared to results obtained from experimental examination of the converters via a laboratory stand. For the purposes of the experimental examination, a control system is developed on the base of a virtual instrument in LabVIEW. The advantages and disadvantages of the different converters are discussed.
\end{abstract}

\section{Introduction}

Resonant DC/DC converters are widely implemented in energy storage systems (ESS) for their soft switching operation and high overall efficiency. Various circuit topologies are used for charging of energy storage elements (ESE), such as supercapacitors and rechargeable batteries, providing either DC current or DC voltage [1, 2].

Due to the specificities of the charging process, the initial charging current may have to be limited via different possible means and methods. Another important issue may be the necessity of galvanic isolation. When higher output power is needed, modular system topology is often used with several converters operating in parallel to a common load [3-6].

The following paperwork considers a resonant $\mathrm{DC} / \mathrm{DC}$ converter with three input inverter stages. Due to the circuit features, its operation principle remains unchanged for larger number of modules. Comparative analysis of the converter operation is carried out for different topologies of the input inverter stages. Model based design is proposed for simplified examination of the complicated processes observed in the separate stages. Models of the examined modules are developed for optimization of the analysis and the design procedure.

\section{Resonant converter circuits with switching capacitor voltage limitation}

The following considered circuits are all derived from the classical resonant inverter half-bridge topology with reverse diodes. As the name suggests, an operation specificity of these converters is the voltage limitation across part of the switching capacitor, which results in a limitation of the supply source current during operation at low resistance loads near the short circuit mode. This feature has a significant contribution to the overall circuit performance in applications for charging of energy storage elements (ESE). Detailed description of the voltage limitation realization and main dependencies is presented in [7].

Another privilege of these converters is their behavior as ideal current sources with respect to the load, which is very useful for obtaining the constant-current ESE charging mode.

\subsection{Resonant inverter with separated input filter capacitor}

Circuit of the resonant inverter with separated input filter capacitor is presented in fig. 1 . It consists of a halfbridge (transistors $T 1$ and $T 2$ ), input filter capacitors $C_{F 1}$ and $C_{F 2}$, resonant tank inductance $L_{K}$, resonant tank capacitor separated into two parts $\left(C_{K}\right.$ and $\left.C_{E}\right)$, limitation diodes $D_{D 1}$ and $D_{D 2}$ and a coupling output transformer $\mathrm{Tr}$. The inverter operation is described in detail in [7].

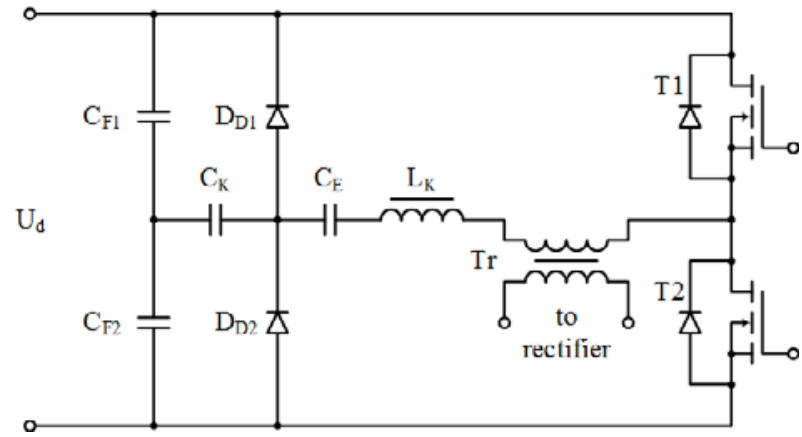

Fig. 1. Circuit of a resonant inverter with switching capacitor voltage limitation and separated input filter capacitor.

\footnotetext{
* Corresponding author: stoyan.vuchev@tu-sofia.bg
} 


\subsection{Resonant inverter with separated switching capacitor}

Another version of the presented in fig. 1 inverter is the circuit with separated switching capacitor (fig. 2). Here, instead of the two large filter capacitors, two equal parts of $\mathrm{C}_{\mathrm{K}}$ are connected directly between the limitation diodes midpoint and the input voltage power poles.

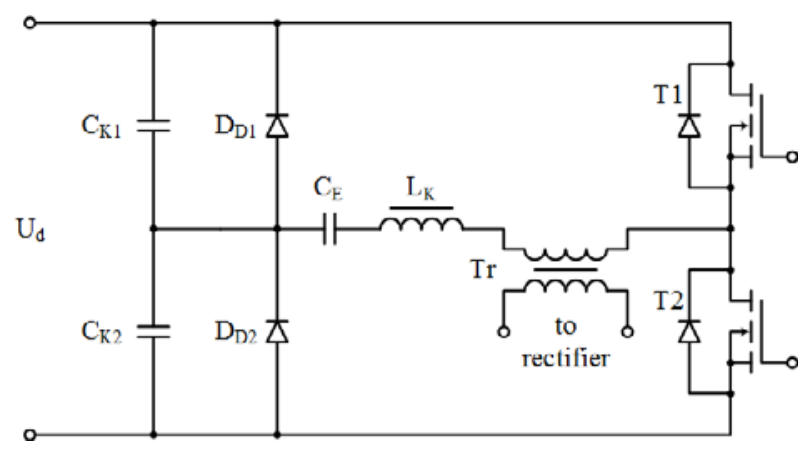

Fig. 2. Circuit of a resonant inverter with voltage limitation across separated switching capacitor.

If the input source is considered as an ideal voltage source with zero resistance, the two capacitor parts are connected in parallel with respect to the AC component. Therefore, each of the two capacitances must be half of the original $\mathrm{C}_{\mathrm{K}}$ value.

$$
C_{K 1}=C_{K 2}=\frac{C_{K}}{2}
$$

Nevertheless, the total installed reactive power of the capacitors remains unchanged, although they share the resonant tank current.

\subsection{Single-stroke resonant inverter circuit}

An equivalent to the presented in fig. 2 circuit with respect to the AC component is the single-stroke resonant inverter (fig. 3). In this case, instead of two equal halves, there is a single $C_{K}$ part of the switching capacitor connected to the input supply negative pole.

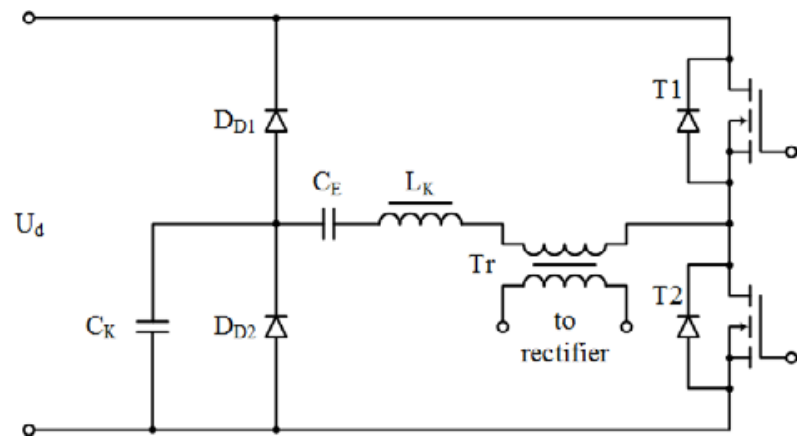

Fig. 3. Circuit of a single-stroke resonant inverter with switching capacitor voltage limitation.

An advantage of the last two inverter topologies is the absence of large input filter capacitors. Moreover, the single-stroke circuit has no capacitor pairs, which eliminates the necessity of two capacitors with well equalized values in order to balance the resonant processes.

\section{Multiphase converter topology}

In order to obtain high output power, a multiphase converter topology is proposed. A generalized blockdiagram of the particular examined three-phase topology is presented in fig. 4. It consists of three inverter stages with coupling transformers, as well as, a three-phase bridge rectifier. The proposed topology allows the number of the input stages to be increased without violating the converter operation - only a proper phase shift of each inverter control signals should be considered.

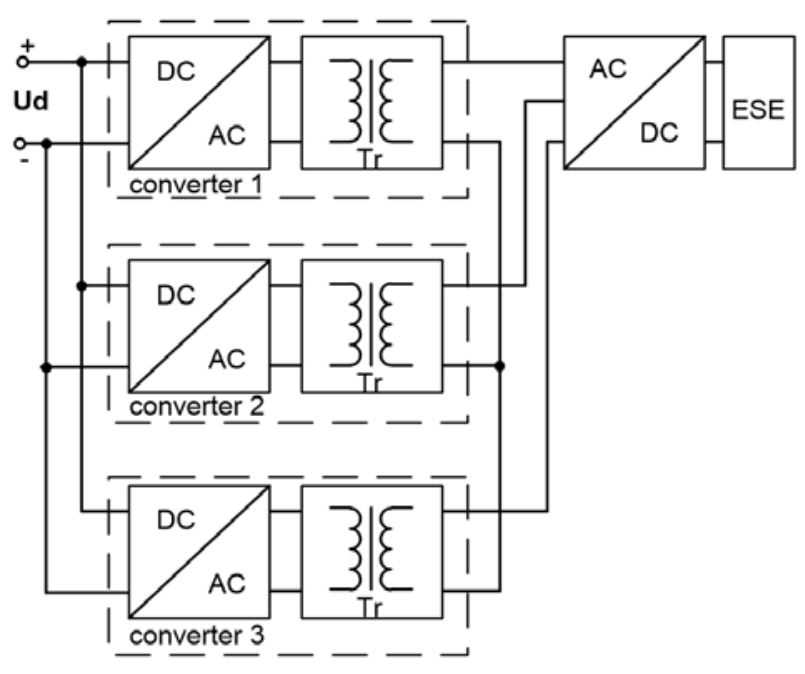

Fig. 4. A generalized block-diagram of the proposed multiphase converter.

Any of the above presented resonant inverter circuits can be implemented for realization of the converter input stages.

\section{Models of the proposed converter}

Models of the proposed multiphase converter are developed on a modular principle in the software environments of MATLAB Simulink and LTspice for the purposes of the circuit examination and the design procedures. This modular principle allows comparative analysis of different circuit topologies for realization of one or more converter modules to be carried out without changing the rest of the model. Thus, one and the same generalized converter model can be used for examination of specific topology parts preserving the operating conditions of the overall model.

The full MATLAB Simulink model of the examined multiphase converter is presented in fig. 5. A detailed description of this model and the separate modules in its composition is presented in [8].

Models of the three above presented resonant inverters with switching capacitor voltage limitation are also developed in MATLAB Simulink in order the circuits to be analyzed and compared during operation as 
input inverter stages of the proposed multiphase converter. The three models are presented in fig. 6 .

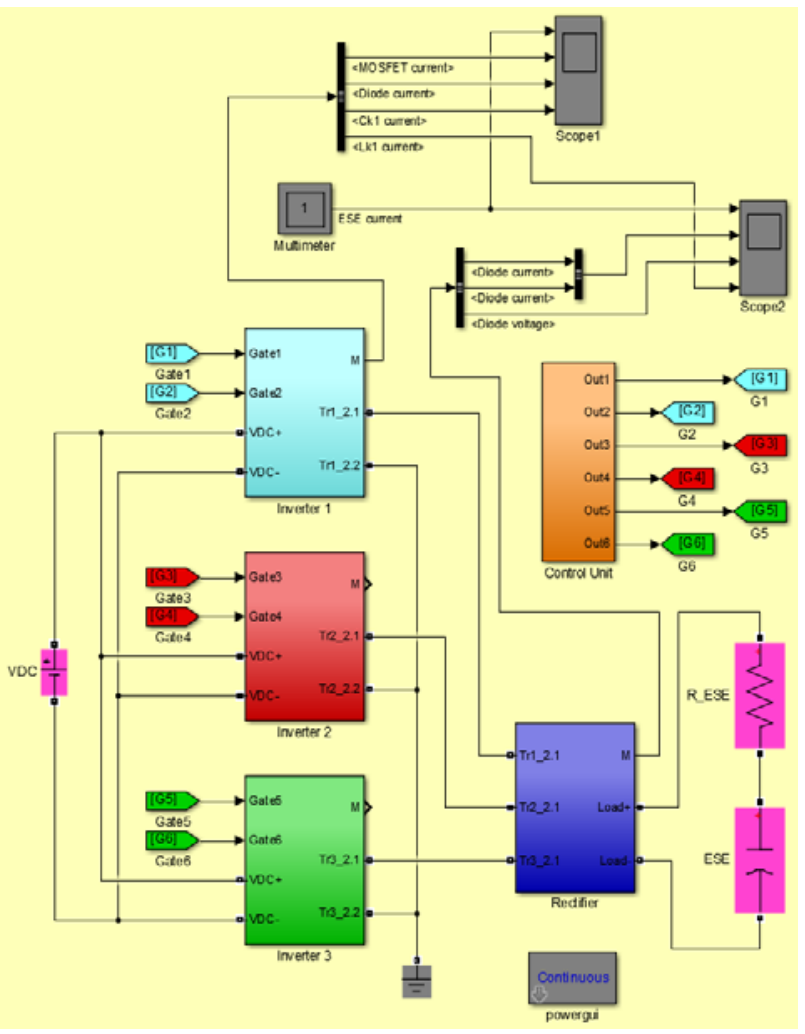

Fig. 5. Full MATLAB Simulink model of the converter.

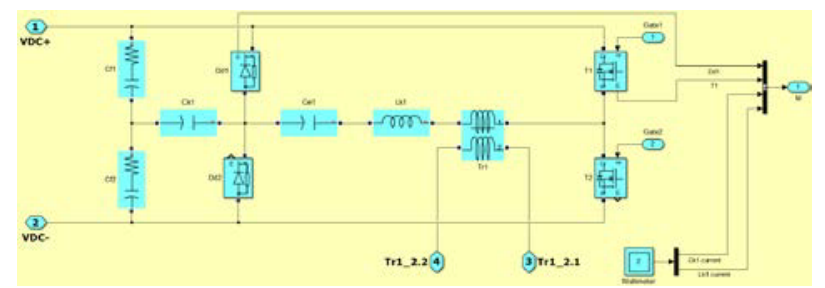

a)

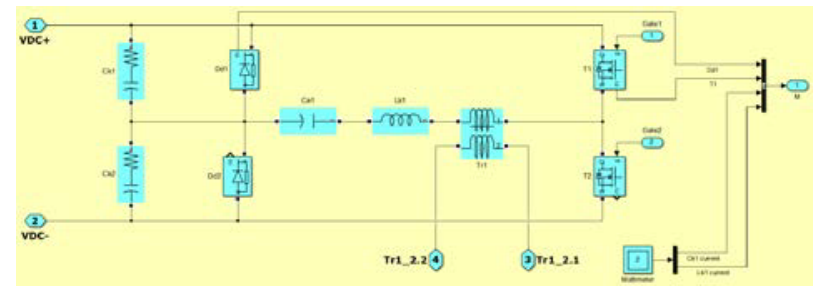

b)

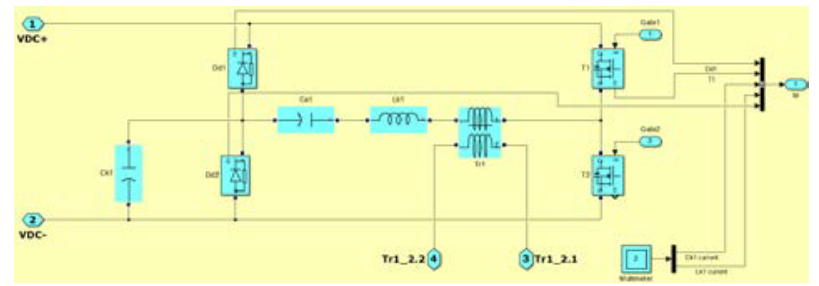

c)

Fig. 6. MATLAB Simulink models of the resonant inverters: a) circuit with separated input filter capacitor; b) circuit with separated switching capacitor; c) single-stroke circuit.

\section{Simulation results}

Results from computer simulations of the proposed multiphase converter are obtained for supply voltage of $30 \mathrm{~V}$, and the ESE is represented by a capacitance of $10 \mathrm{mF}$ and a resistance of $1 \Omega$ (including parasitic resistance of connections). The transformer ratio is 1 . The other circuit values are as follows:

$$
C_{K}=2.8 \mu F, C_{E}=0.4 \mu F, L_{K}=135 \mu H
$$

\subsection{MATLAB simulation results}

The results obtained from computer simulations with the developed in MATLAB Simulink model of the proposed converter with single-stroke resonant inverter stages are presented in fig. 7, fig. 8 and fig. 9. The obtained waveforms are identical for the three examined input inverter topologies. The only difference is the maximum value of the $C_{K}$ current which is twice smaller for the circuit with separated switching capacitors.

For the purposes of the present investigation, the capacitance representing the ESE is significantly reduced in order to obtain proper illustration of the whole charging process for reasonably small simulation times.

As it can be observed, the limitation diode interval of conduction strongly depends on the ESE state of charge. For lower values of the load voltage, the voltage limitation across $C_{K}$ is significant, which results in limitation of the initial charging current. With increase of the ESE voltage, smaller limitation is applied.

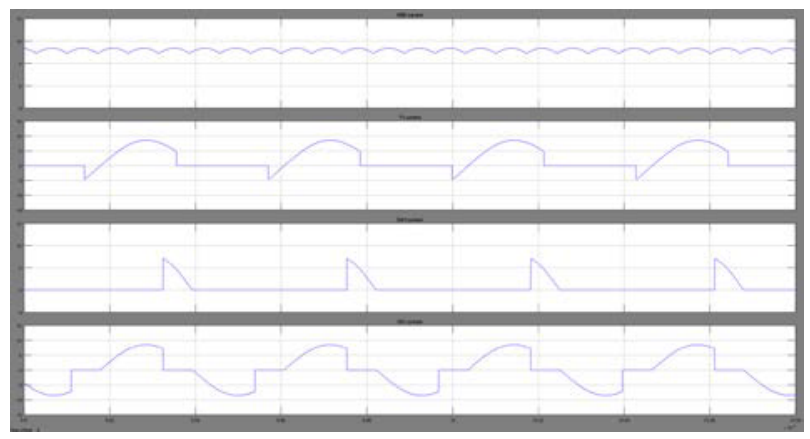

Fig. 7. Waveforms of the ESE, the transistor T1, the limitation diode $D_{D 1}$ and the switching capacitor $C_{K 1}$ currents at $10 \mathrm{~ms}$ simulation time (MATLAB Simulink model).

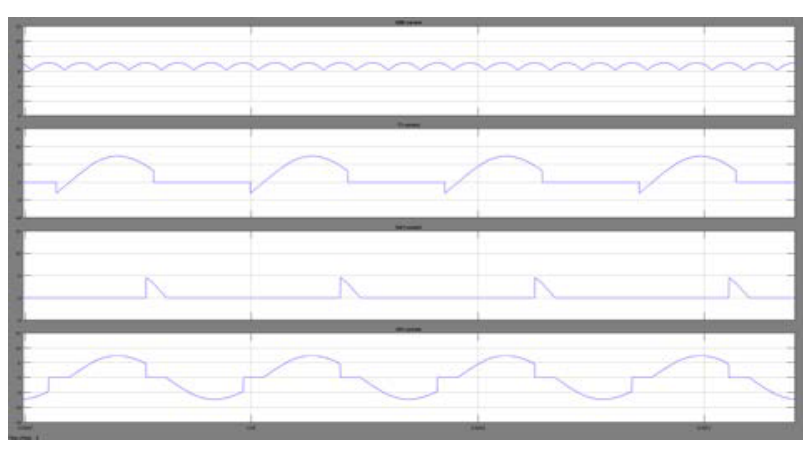

Fig. 8. Waveforms of the ESE, the transistor T1, the limitation diode $D_{D 1}$ and the switching capacitor $C_{K 1}$ currents at $20 \mathrm{~ms}$ simulation time (MATLAB Simulink model). 


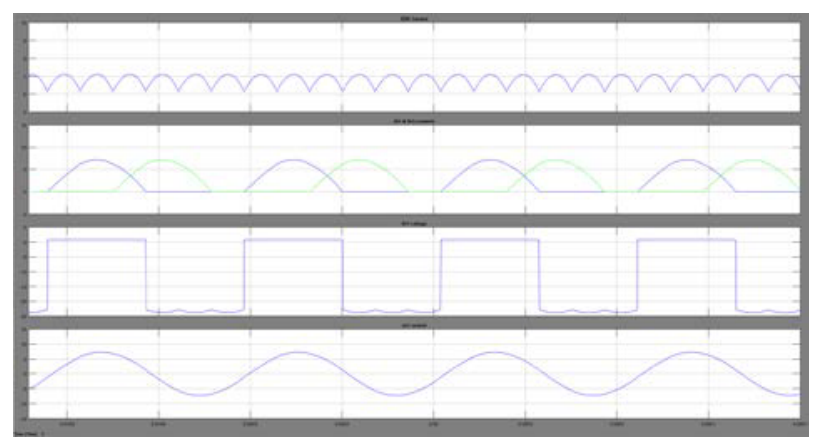

Fig. 9. Waveforms of the ESE current, the rectifier diodes $D_{R 1}$ and $D_{R 3}$ currents, the diode $D_{R 1}$ voltage and the resonant inductance $\mathrm{L}_{\mathrm{K} 1}$ current at $20 \mathrm{~ms}$ simulation time (MATLAB Simulink model).

\subsection{LTspice simulation results}

For verification of the obtained simulation results from the model of the proposed multiphase converter in MATLAB Simulink, an identical model is developed in the software environment of LTspice. During the simulation examination, the same circuit element values are used.

Again, the capacitance representing the ESE is significantly reduced in order to obtain proper illustration of the whole charging process for reasonably small simulation times.

The results obtained from the LTspice model of the proposed multiphase converter are presented in fig. 10, fig. 11 and fig. 12. As it can be observed, they are identical to those from the MATLAB Simulink model, which verifies both their authenticity.

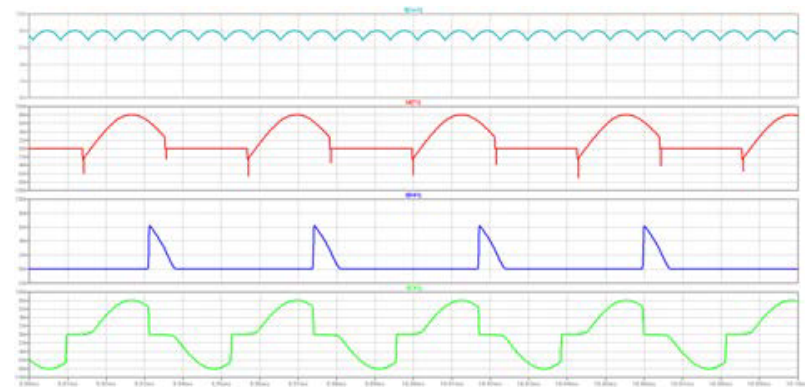

Fig. 10. Waveforms of the ESE, the transistor T1, the limitation diode $\mathrm{D}_{\mathrm{D} 1}$ and the switching capacitor $\mathrm{C}_{\mathrm{K} 1}$ currents at $10 \mathrm{~ms}$ simulation time (LTspice model).

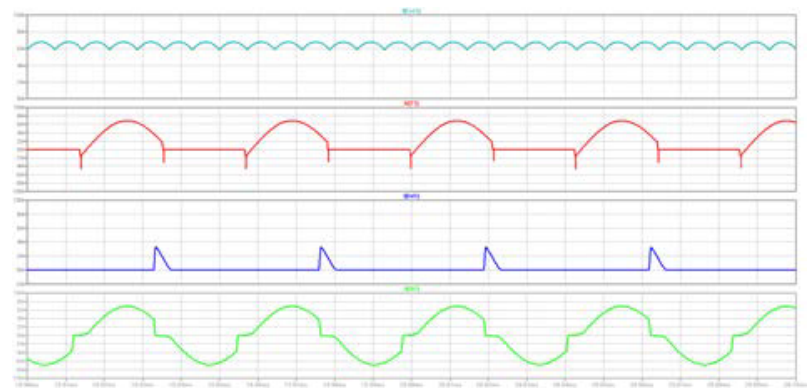

Fig. 11. Waveforms of the ESE, the transistor T1, the limitation diode $\mathrm{D}_{\mathrm{D} 1}$ and the switching capacitor $\mathrm{C}_{\mathrm{K} 1}$ currents at $20 \mathrm{~ms}$ simulation time (LTspice model).

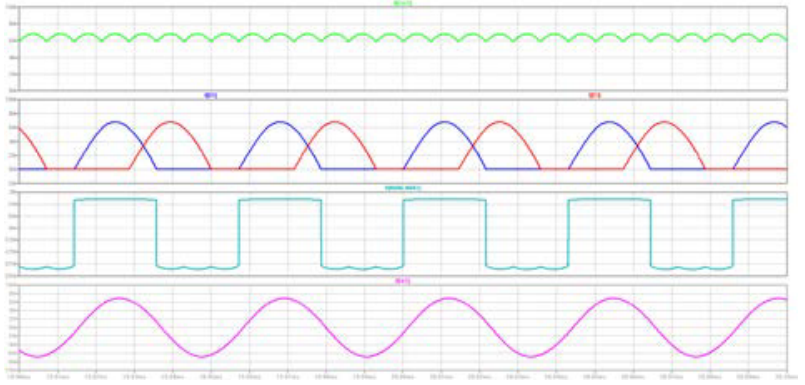

Fig. 12. Waveforms of the ESE current, the rectifier diodes $D_{R 1}$ and $D_{R 3}$ currents, the diode $D_{R 1}$ voltage and the resonant inductance $\mathrm{L}_{\mathrm{K} 1}$ current at $20 \mathrm{~ms}$ simulation time (LTspice model).

\subsection{Tolerances of the circuit elements}

The tolerances of the elements in the examined circuits play an important role in the whole converter performance. Deviation in one or more of the resonant tank values results in a different resonant frequency, i.e. different detuning for a fixed operating frequency. As a result, lack of balance between the separate stage currents occurs.

The above can be observed in fig. 13 where simulation results from the MATLAB Simulink converter model are presented for a changed value of one of the resonant tank inductors $\left(L_{K 1}=155 \mu H\right)$. The other circuit component values are unchanged.

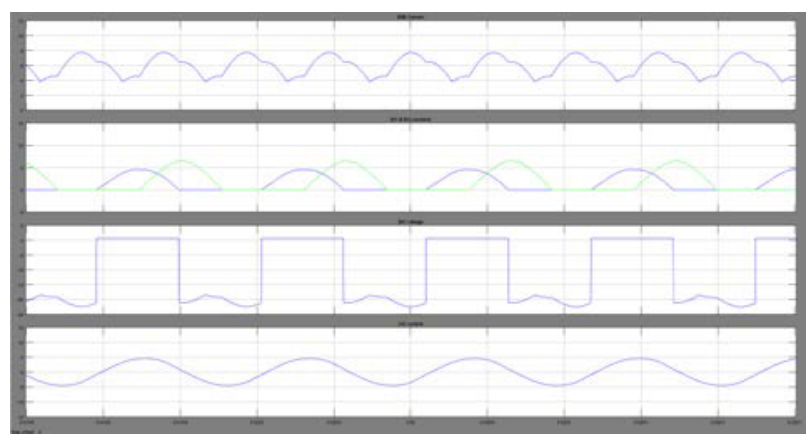

Fig. 13. Waveforms of the ESE, the transistor $T 1$, the limitation diode $\mathrm{D}_{\mathrm{D} 1}$ and the switching capacitor $\mathrm{C}_{\mathrm{K} 1}$ currents at $20 \mathrm{~ms}$ simulation time (MATLAB Simulink model with circuit imbalance).

An identical simulation examination is carried out in LTspice for verification of the obtained results (fig. 14).

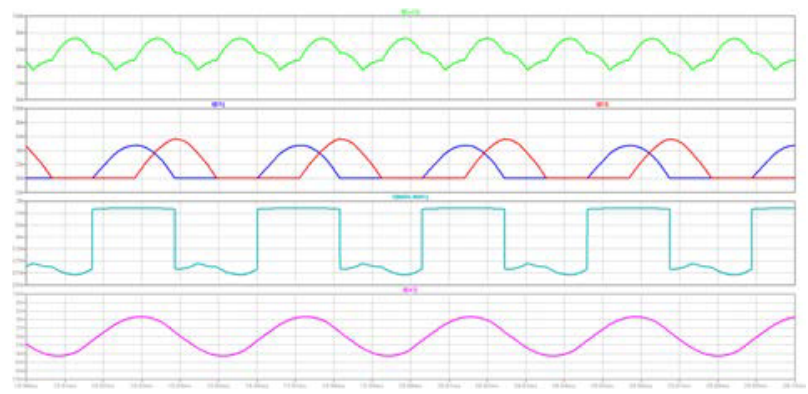

Fig. 14. Waveforms of the ESE, the transistor $T 1$, the limitation diode $D_{D 1}$ and the switching capacitor $C_{K 1}$ currents at $20 \mathrm{~ms}$ simulation time (LTspice model with circuit imbalance). 


\section{Experimental examinations}

For further verification of the obtained simulation results, experimental examinations of the proposed multiphase converter based on single-stroke resonant inverter input modules are carried out on a laboratory stand. The presented waveforms (fig. 15 to fig. 18) correspond to the charging process of a $58 \mathrm{~F} / 16 \mathrm{~V}$ supercapacitor. The other circuit parameter values are identical to the ones from the two simulation models.

The experimentally obtained current waveforms present the operation of an imbalanced converter due to the tolerances of the circuit elements. As a result of these tolerances, an imbalance between the separate inverter stage currents occurs. This can easily be observed from the non-symmetric ESE current waveform, as well as, from the rectifier diode current waveforms having different maximum values.

The presented waveforms of the currents through the diodes, the transistors and the $\mathrm{L}_{\mathrm{K}}$ inductors are obtained via a current probe with ratio of $10 \mathrm{~mA} / \mathrm{mV}$. The waveform of the ESE current is obtained via a measurement shunt.

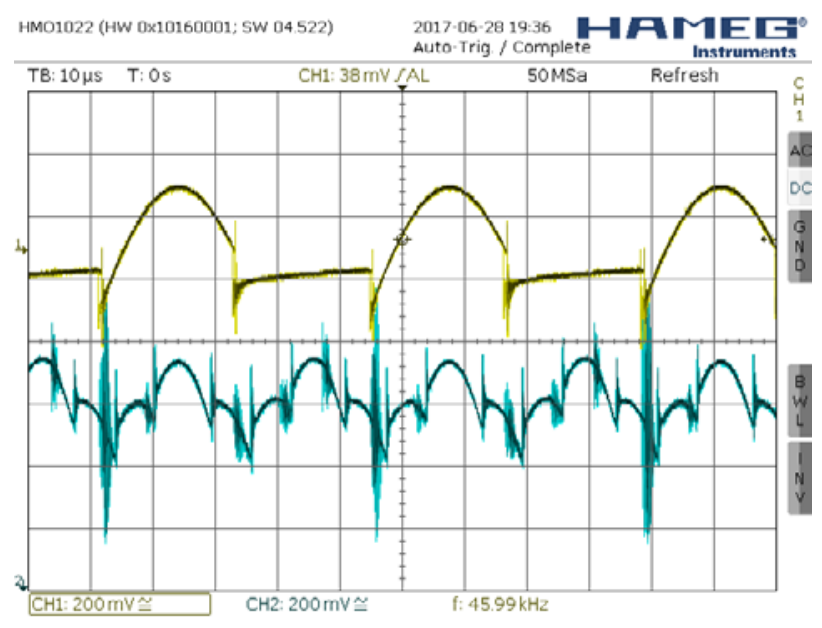

Fig. 15. Waveforms of the transistor $T 1$ and the ESE currents.

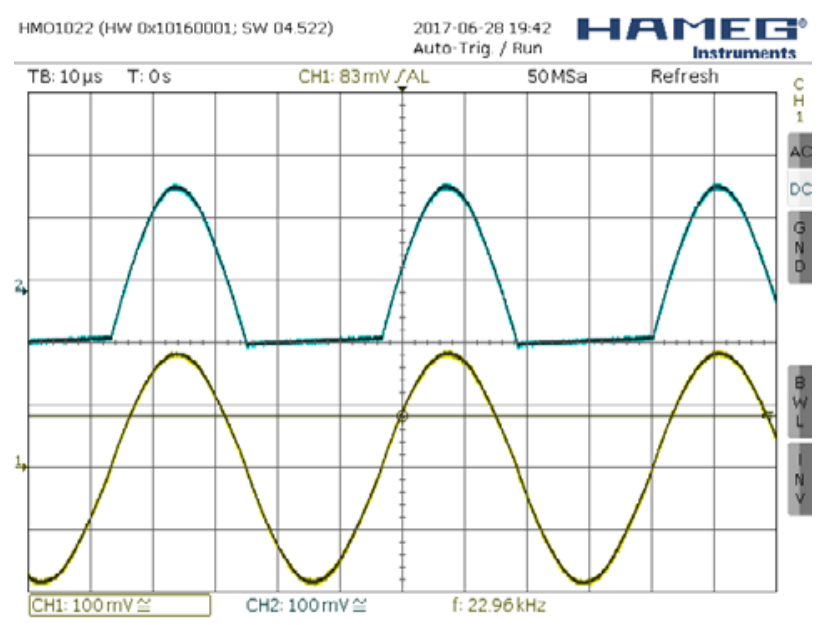

Fig. 16. Waveforms of the diode $D_{R 1}$ and the $L_{K 1}$ currents.

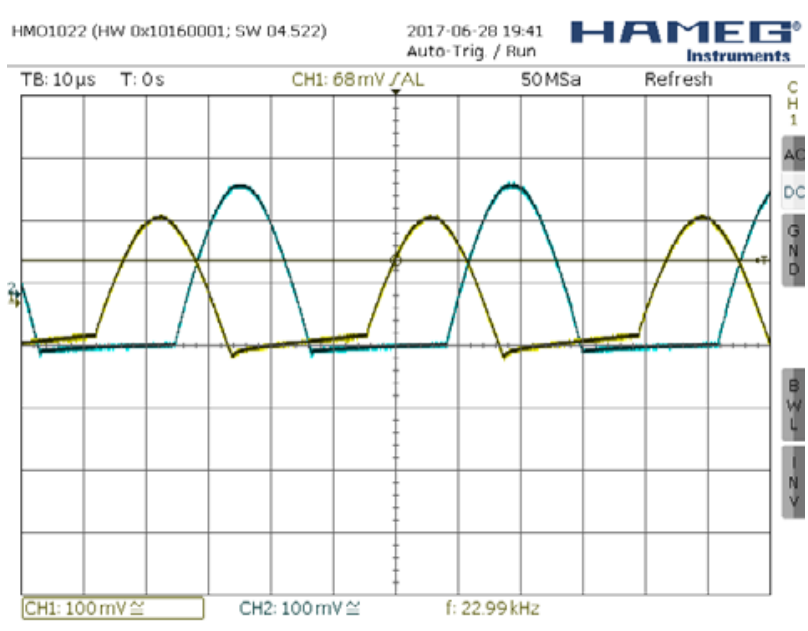

Fig. 17. Waveforms of the diodes $D_{R 1}$ and $D_{R 3}$ currents.

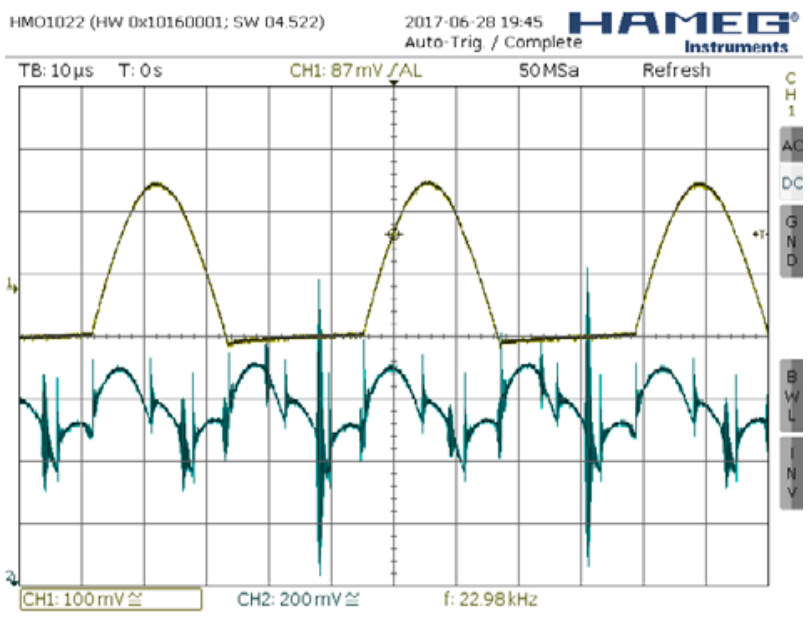

Fig. 18. Waveforms of the diodes $D_{R 1}$ and the ESE currents.

\section{Conclusion}

An investigation of a multiphase converter for charging of ESE is carried out. Different topologies of resonant inverters with switching capacitor voltage limitation are considered for the converter realization. These stages operate at specific load.

Another specialty is the rectifier operation with two diodes from the same group (anode or cathode) operating simultaneously during part of the time as the stage operates in current rectification mode. This can be observed from the waveforms presented in fig. 9, 12, 13, 14 and 17.

With variation of the resonant tank element values, a stage operating mode is changed with respect to those of the other modules. Moreover, this change causes different changes in the operating modes of the other two stages although they have identical component values.

By the means of the developed multiphase converters simulation models, the influence of the component values variations on the separate stages operating modes can be examined. The mutual influences can change the operating modes, and, in some cases, deviation from the limitation mode may occur. Therefore, in order to 
minimize these influences, topologies with least possible number of coupled reactive elements (capacitors) are necessary to be used.

The developed models allow parametric analysis to be carried out. Because of the complexity of the circuit processes and the lack of steady state operation during ESE charging, analytical expressions for the circuit currents and voltages are difficult to be obtained. For design of such converters model based design is used. In the developed model, the converter is based on a modular structure consisting of separate sub-circuits, which favors the comparative analysis for a constant configuration with different basic converter circuits. Obtaining different operating modes of the multiphase converter allows to evaluate in which cases a synchronous rectifier realization is possible in order to increase the converter efficiency.

The carried out research is realized in the frames of project BG05M2OP001-2.009-0033, Operational Program Science and Education for Smart Growth 20142020.

\section{References}

1. Cabrane, Z., M. Ouassaid, M. Maaroufi, WCCS Proceedings, pp. 380-385 (2014).

2. Madzharov, N., Journal of Engineering Science and Technology Review, 9, 6 (2016).

3. Cornea, O., N. Muntean, M. Gavris, OPTIM 2010, pp. 589-594 (2010).

4. Zarkov, Z., I. Bachev, L. Stoyanov, V. Lazarov, IEEE-PEMC 2016 Proceedings, pp. 32-37 (2016).

5. Kanchev, H., F. Colas, V. Lazarov, B. Francois, IEEE Transactions on Sustainable Energy, 5, 4 (2014).

6. Antoszczuk, P., R. Retegui, M. Funes, IEEE Transactions on Industrial Informatics, 10, 4, pp. 2224-2232 (2014).

7. Arnaudov, D., N. Hinov, I. Nedyalkov, ELEKTROTECHNICA \& ELEKTRONICA E+E, 50, pp. 11-18 (2015).

8. Arnaudov, D., N. Hinov, S. Vuchev, I. Nedyalkov, PCIM Europe 2017 Proceedings, pp. 1130-1136 (2017). 